\title{
Information Theory of Evolutionary Stages in Noninteger Dimensional Spaces
}

\author{
Subhash Kak \\ Oklahoma State University, Stillwater
}

\begin{abstract}
This paper investigates evolution of a physical system through intermediate noninteger dimensions to provide a phenomenological explanation for the system's emergent properties. In recent papers it was shown that physical space is associated with noninteger dimensionality and its value is associated with the strength of attractive inverse square law and this has applications to diverse fields including the design of metamaterials. Here this information-theoretic analysis is applied to cosmology to yields a novel noninteger dimensional explanation for filaments and sheets of matter, inflation, and the accelerating expansion of the universe, without the need to postulate inflation field or dark energy as the drivers of this expansion. Furthermore, the analysis shown that in the future as the zerodimension residual potential declines further, the expansion will slow and then reverse. Evolution across noninteger spaces has potential relevance for the study of materials that emerge from compressing three-dimensional volumes into lower dimensions.
\end{abstract}

\section{Introduction}

There are two intertwined aspects to our conception of reality: physical (matter and energy together with their distribution in various forms), and informational. Their mutual interconnection comes from the fact that we know about the physical world through information [1]. Science is concerned with enlarging the physical view to the extent it is possible, but in recent years the spectacular successes of physical theories have hit roadblocks in the inability to construct grand unified theories to bring together the Standard Model and the strong force, and to find a place for gravity in these theories. Furthermore, the accelerating expansion of the universe that began 4 billion years ago, based on supernovae that provide good standard candles, is now being explained in terms of dark energy for which no evidence exists. Since hypothetical dark matter and dark energy are now required to describe $96 \%$ of the physical universe [2][3], it becomes imperative to go back and examine the assumptions related to information on which current theories are based.

In a recent work, it was proposed that physical space in its most information-efficient form has a dimensionality of $e$ and this helps resolve the problem of Hubble tension 
[4]. In a subsequent paper [5], it was shown that the phenomenon of asymptotic freedom, in which there is weak interaction at high energies and strong interaction at low energies as in the case of quarks that constitute a proton, can also be explained within the paradigm of noninteger dimensionality [6][7]. It was also argued that the inverse square law is a consequence and was shown that the potential is zero for all values between 1 and 2 and it peaks at $d_{\text {crit }}$, a value between 2 and 3 . It was also argued that these ideas apply directly to cosmology. The previous results are motivation to push further with the information-centered view of physical reality and investigate its strengths and limitations. Observations of the cosmic microwave background radiation and the scale-invariant distribution of structures [8][9] indicate that the spatial variations in energy in the early universe were nearly scale-invariant. Such scale-invariance means noninteger dimensionality and, therefore, it is further reason to consider noninteger dimensionality during the evolutionary phase.

In the present paper, the evolution of the universe is investigated and it is shown that the presence of filaments and sheets, inflation, slowing and subsequent accelerated expansion of the universe flow naturally from our model, obviating the need to postulate dark energy.

\section{Dimensionality energy}

Our thesis is that since e-dimensionality is optimal for representations [10][11] it must include physical space [4][5], and several issues with this have been investigated [12][13]. The potential of space in its zero-dimensional form may be called $V_{0}$ and its value may be fixed from experimental observations. This potential eventually transforms the dimensionality of space, which increases in value from 0 to the optimal $e$, with accompanying transformations into radiation and increasingly complex forms of matter and clumped structures.

The metric for a flat expanding universe with scale factor $a(t)$, with $t$ as the cosmic or physical time, has the form $d s^{2}=-c^{2} d t^{2}+a^{2}(t)\left(d x^{2}+d y^{2}+d z^{2}\right)$. For the universe to satisfy the cosmological principle, that is it is both homogeneous and isotropic it is essential that as dimensional potential $V(t)$ changes into radiation and baryonic matter, the size of the universe should increase in proportion to the amount of matter that is being created.

The evolution of the universe must be viewed in its dynamics with respect to $d$, which, in turn, creates cosmological time as the dynamic variable, $V$, evolves. The 
measured Hubble expansion parameter $H(t)=\frac{\dot{a}}{a}=\frac{\dot{R}}{R}$ is positive if the universe is expanding and negative if it is contracting and $R$ is the scale factor.

The potential as a function of the scalar field $\phi$ should be $V(\phi)=\frac{m^{2}}{2} \phi^{2}$ but due to the expansion of the universe we need to use the damped harmonic oscillator equation for the field:

$$
\ddot{\phi}+3 H \dot{\phi}=-m^{2} \phi
$$

It is reasonable to assume that the rate of change of this potential is proportional in a negative to the potential itself. In other words,

$$
\frac{d V}{d t}=-\alpha V
$$

This corresponds to the solution

$$
V(t)=V_{0} e^{-\alpha t}
$$

If matter increases in inverse proportion to the rate at which $V(t)$ is changing, that is $m(t)=m_{0} e^{\alpha t}$, then the cosmological principle requires that the size of the universe increases by the same factor. In other words, the size of the universe at time $t$ compared to an earlier reference epoch is:

$$
a(t)=a_{0} e^{\alpha t}
$$

There is an additional complicating factor. As space dimensionality increases, it transforms first into radiation energy and subsequently into matter, which is associated with an increasing stronger attractive force that begins to counter the expansion. The interplay between these two first slows down the expansion from its initial rate and later as the attractive force begins to weaken, the expansion accelerates. Therefore, the rate of expansion must be revised to take into consideration the attractive field associated with matter. This will be discussed further in the next section, but before that we sketch the standard view, which speaks of five epochs of evolution. These are [14][15][16[17]]:

1) Inflation with a near instantaneous expansion from the initial state which is scalar field dominated; 2) Hot big bang that is radiation dominated; 3) Matter dominated expansion from its initial rapid rate that continued for nearly 9.8 billion years ago; 4 ) Accelerating expansion of the universe that began about 4 billion years ago; and 5 ) 
Future states that can be described only after proper explanation for the acceleration expansion has been found.

Consider the scalar field $\phi(t)$ for a homogeneous universe, then we can combine the Einstein equation

$$
H^{2}+\frac{k}{a^{2}}=\frac{1}{6}\left(\dot{\phi}^{2}+m^{2} \phi^{2}\right)
$$

with equation (1) and consider the initial case where $\ddot{\phi} \ll \dot{\phi}$, so that $3 H \dot{\phi}=-m^{2}$; and since the resistance to the field is low, consequently $a$ is large, and the change in the field is small. Therefore, $H^{2}=\frac{1}{6} m^{2} \phi^{2}$, where $H=\frac{1}{\sqrt{6}} m \phi$.

In the hot big bang [18] the scaling is $a(t)=a_{0} t^{1 / 2}$ in which baryosynthesis, neutrino decoupling, and nucleosynthesis take place. The next epoch has $a(t)=$ $a_{0} t^{2 / 3}$, but the gravitational dynamics hinge on pressure-free cold dark matter (CDM) for which also no evidence has yet been found.

In the $\Lambda C D M$ model [19], the Friedmann equations define how the energy in the universe drives expansion by relating the gravitational constant $G$ to the energy density $\rho c^{2}, p$ pressure, and $k$ a dimensionless constant for the curvature of the universe and the cosmological constant $\Lambda$ to describe the accelerating expansion of the universe by means of the field $\varphi$, which represents dark energy, such that $\rho_{\varphi}+$ $\frac{3 p_{\varphi}}{c^{2}}<0$ :

$$
\begin{aligned}
& H^{2}=\frac{8 \pi G}{3} \rho-\frac{k c^{2}}{R^{2}}+\frac{\Lambda c^{2}}{3} \\
& \frac{\ddot{a}}{a}=-\frac{4}{3} \pi G\left(\rho+\frac{3 p}{c^{2}}\right)+\frac{\Lambda c^{2}}{3}
\end{aligned}
$$

These equations don't remain valid if $G$ varies [20][21]. A description with a varying gravitational constant can be designed to satisfy the equivalence principle in its weak form but not in its strong form. The difficulty with the standard view is that there is no observational evidence for the dark energy fields as well as CDM. Furthermore, it ignores the noninteger aspects of space dimensionality.

\section{Variation of $\mathrm{G}$ with changing $d$}

For noninteger dimensionality spaces, the previous paper [5] presented the potential $p(d, r)$ at a distance $r$ from an object of unit mass as a function of the dimensionality 
$d$ of the space and function $f$ related to the difference between the actual dimension of the space and its ceiling integer value:

$$
p(d, r)= \begin{cases}\frac{f(2-d)}{4 \pi}, & 1 \leq d \leq 2 \\ \frac{f(3-d)}{4 \pi r d}, & 2 \leq d \leq 3\end{cases}
$$

For $1 \leq d \leq 2$, the potential is independent of $r$ and therefore it may be taken to be equal zero. For, $2 \leq d \leq 3$, the potential corresponds to the inverse square law which we may on account of the Occam's razor take to be the same as the gravitational force. As explained in the previous paper, the function $f(3-d)$ is zero for integer values.

For $0 \leq d \leq 1$, we need to know $f(1-d)$. Given that the potential $1 \leq d \leq$ 2 turns out to be zero, we may assume that $f(2-d)$ is zero and by extension take $f(1-d)$ to be zero also. Plugging that into $p(d, r)$ is $\frac{f(1-d) d r}{s_{[d]}(d, r)}$ for $0 \leq d \leq 1$, we have the case of $0 / 0$ which is indeterminate as we don't have further information to simplify it directly. But in extension with the situation for $1 \leq d \leq 2$, we will take

$$
p(d, r)=0,0 \leq d \leq 1
$$

As explained in the previous paper, the simplest choice for $f(3-d)$ is

$$
f_{A}(3-d)=(d-2)(3-d) \frac{\ln d}{d}
$$

This has a peak at $d_{\text {crit }}=2.455$. In other word, the gravitational force will be maximum at this value of dimensionality. If on the other hand, we didn't consider the normalizing weight $\frac{\ln d}{d}$ in the above expression, then the force will be maximum at $d_{\text {crit }}=2.5$. We assume that this latter value is appropriate for further discussion of the question.

Thus equations (9) and (10) represent the gravitational potential associated with different dimensions in Figure 1.

The attractive potential at a unit distance for unit mass will be a function of $d$ for $2 \leq$ $d \leq 3$, and it may be written as:

$$
V_{g}(d)=G\left(5 d-d^{2}-6\right)
$$




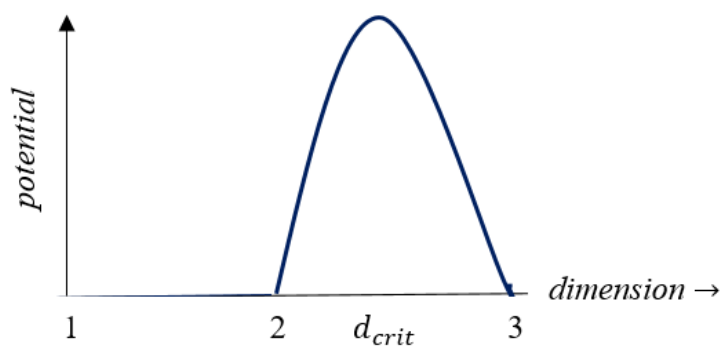

Figure 1. The potential map for $1 \leq d \leq 3$

If it is assumed that the evolution of dimensionality is uniform with respect to time until it reaches the optimal value of $e$, the gravitational constant will have the functional form (Figure 2):

$$
G(d, t)=G(d)= \begin{cases}G_{0}\left(5 d-d^{2}-6\right) ; & d \leq e \\ 0.202 G_{0} ; & d>e\end{cases}
$$

in units of universal time and $G_{0}$ is its reference value.

We find that $G(d)_{\max }=0.25$ in reference units, whereas $G(d=e)=0.202$ in the same units. This means that the gravitational attraction has weakened from its peak value by the factor

$$
\frac{G(e)}{G(d)_{\max }}=0.8094
$$

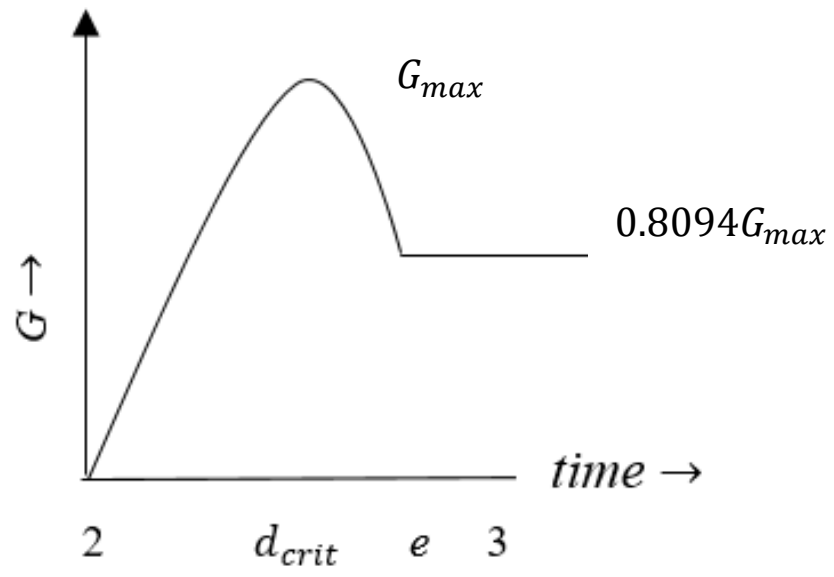

Figure 2. Time variation of $\mathrm{G}$ 
To consider the expansion rate, we must look at the difference between (1) and (9):

$$
V(t)-V_{g}(t)=V_{0} e^{-\alpha t}-G(t)
$$

The accelerated expansion of the universe began about 4 billion years ago [21][22], so this turning point should be close to when the gravitational constant $G$ peaked and began to weaken thereafter. It may not be exactly this turning point because the expansion would depend on the relative strengths of the two potentials and, therefore, may have occurred somewhat later.

\section{Evolutionary epochs}

Now we consider the question of investigating the evolution of $d$ with respect to time. It is assumed that the dimensions expanded in response to the initial potential associated with dimension 0 ; it follows that it will grow into increasing dimensionality on its way to the optimal value of $e$. The interplay of time-varying gravitation and the decaying field $V$, creates four epochs in the evolution of the universe. We will see these in terms of different ranges of $d$.

From its initial point singularity, the universe must increase its dimensionality in stages. First, the initial dimensionality potential transforms into radiation and subsequently to matter. This means that it must first become filaments $(d=1)$ or line fractals that are one-dimensional scale-invariant structures and then sheets $(d=2)$ [23] before transforming into matter as the dimensionality increases beyond 2 . One may speculate that the filaments consisted of neutrinos and photons came later.

As the dimensionality becomes greater than 2 , there will be an exponential rise over the values $2 \leq d \leq e$. Beyond 2 , the inverse square law arises and by Occam's Razor we may take it to be gravitation.

Now we return to our evolutionary epochs:

Epoch 1. Filaments. $0 \leq d \leq 1$.

We already provided reasons to assume that the potential associated with the filaments is zero. This epoch is characterized by single-dimensional fractal filaments with optimal value reaching to $e / 3$.

Epoch 2. Inflationary expansion, $1 \leq d \leq 2$. Sheets.

The drop in the potential during this expansion is also zero, because the attractive force is independent of separation. During $1 \leq d \leq 2$, fractals sheets will eventually 
rise to the value $2 e / 3$. The radiation energy will get distributed in a scale-invariant manner over the sheet. Other forces may be associated with this range. The expansion will be near instantantanous.

If the initial expansions are in terms of neutrinos, then we know the interaction amongst them will be very small but it will not create an infinitely large space of filaments and sheets.

Epoch 3. Radiation to matter $2 \leq d \leq d_{\text {crit }}$ Slowing expansion.

As the dimensionality becomes greater than 2, the inverse square law arises and by Occam's Razor we may take it to be gravitation. Since the attractive force increases for $2 \leq d \leq d_{\text {crit }}$, there will be slowing down of the expansion over this range for matter is pulled towards each other with increasing value. It is indeed reasonable to assume that it has two sub-epochs: one in which radiation dominates, and the other in which matter dominates. But after $d_{\text {crit }}$, that appears to occur somewhere in the middle of 2 and 3 (we have, for simplicity taken it to be 2.5) the gravitation attraction decreases and, therefore, the expansion rate will increase. This is shown in Figure 3.

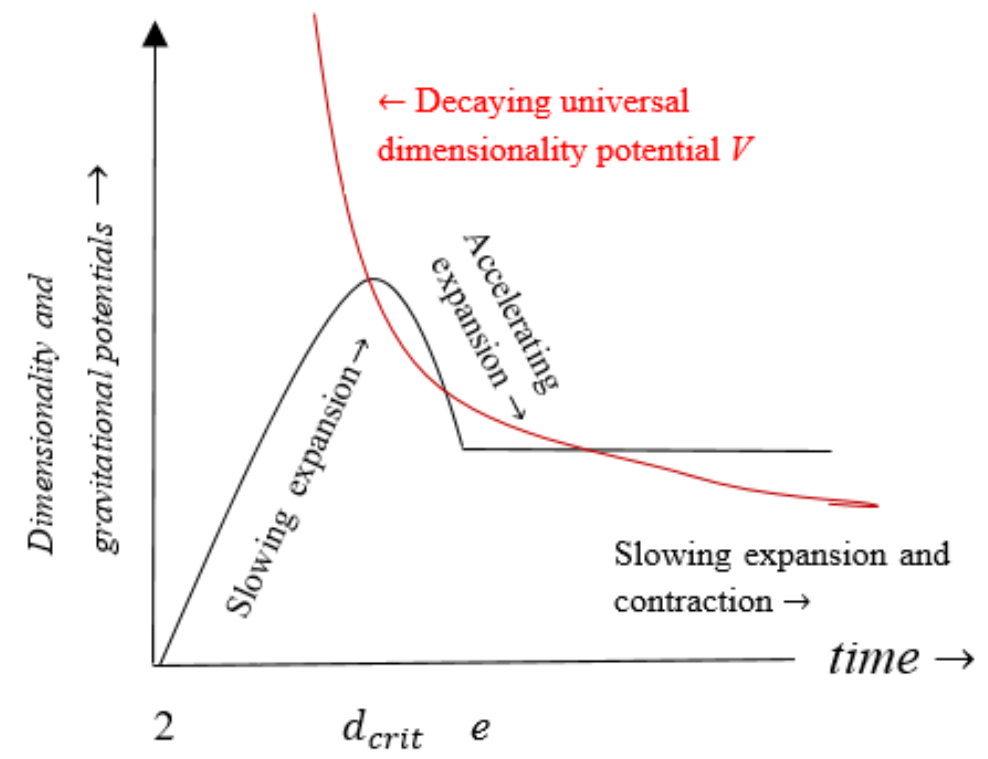

Figure 3. The stages of inflation, slowing expansion, and accelerated expansion

Epoch 4. Accelerating expansion. $d_{\text {crit }} \leq d \leq e$

A much slower and gradual expansion of space continued after this, until at around 9.8 billion years ( 4 billion years ago) it began to gradually expand more quickly, and is still doing so. There is no need to postulate the existence of dark energy appearing 
as a cosmological constant in the simplest gravitational models as a way to explain this late-time acceleration.

Epoch 5. The future $d=e$ with decreasing $V$.

This will lead first to slowing expansion and subsequently to contraction.

\section{Conclusions}

The postulation of dimensional energy and the big bang as a result of the "explosion" of $d=0$ space into higher dimensions with the evolution provides an alternative to the current models of cosmology.

This alternative model shows that quite like in the established theory, the expansion goes through different stages. These are a very rapid expansion at a nearly instantaneous rate quite like the inflation of standard theory, followed by an inversesquare law (gravity) attraction mode with two sub-phases (radiation-dominant and matter-dominant) where this attraction becomes increasing larger which slows down the expansion from its initial phase, accelerated expansion as the attraction force declines and gravitation holds steady. This model predicts that in the future the potential $\mathrm{V}$ will progressively decline and at a certain point it will be less than that of gravitational attraction; it will thus be characterized by slowing expansion followed eventually by contraction of the universe.

Our model shows that there is no need to postulate dark energy as being responsible for the accelerated expansion, for in our theory it was a consequence of the weakening of the attractive gravitational force as compared to the dimension-driven energy responsible for the expansion of the universe.

The potential tests for this theory is that it should be able to explain all observational phenomena without invoking dark matter and dark energy. Furthermore, evidence should be found for changing gravitational field in the past.

The notion of dimension applies to physical reality at all conceivable scales, therefore one must also consider anomalous mechanical properties of materials that emerge from compressing three-dimensional volumes into lower dimensions, which may be seen as an example of increasing the energy scale, with prospective applications to superconductivity, ferroelectricity, information communication, sensing and detection. 


\section{REFERENCES}

1. Kak, S. Logic of representation and information. (2021) Preprint at https://www.techrxiv.org/articles/preprint/Logic of Representation and Info rmation/13601939

2. Frieman, J. A., Turner, M. S., Huterer, D. Dark energy and the accelerating universe. Annual Review of Astronomy and Astrophysics. 46 (1): 385-432 (2008)

3. Uzan, J.-P. The big-bang theory: construction, evolution and status. Seminaire Poincare 20, 1 - 69 (2015)

4. Kak, S. Information theory and dimensionality of space. Scientific Reports 10, 20733 (2020). https://doi.org/10.1038/s41598-020-77855-9

5. Kak, S. Asymptotic freedom and noninteger dimensionality. Scientific Reports 11, (2021).

6. Schleicher, D. Hausdorff dimension, its properties, and its surprises. The American Mathematical Monthly. 114 (6), 509-52 (2007)

7. Stillinger, F.H. Axiomatic basis for spaces with noninteger dimensions. Journal of Mathematical Physics 18, 1224- 1234 (1977).

8. Durrer R (2008). The Cosmic Microwave Background. Cambridge: Cambridge University Press.

9. Joyce, M., Labini, F.S., Gabrielli, A., Montouri, M.. Pietronero, L. Basic Properties of Galaxy Clustering in the light of recent results from the Sloan Digital Sky Survey. Astronomy and Astrophysics. 443 (11): 11-16 (2005)

10. Kak, S. The base-e representation of numbers and the power law. Circuits Syst. Signal Process. (2020) https://doi.org/10.1007/s00034-020-01480-0

11. Kak, S. The intrinsic dimensionality of data. Circuits Syst. Signal Process. (2020); https://doi.org/10.1007/s00034-020-01583-8

12. Kak, S. Information, representation, and structure. International Conference on Recent Trends in Mathematics and Its Applications to Graphs, Networks and Petri Nets, New Delhi, India (2020).

13. Kak, S. Fractals with optimal information dimension. (2020) Preprint at https://www.techrxiv.org/articles/preprint/Fractals_with_optimal_informatio n_dimension/13369598

14. Peebles, P. J. E., Ratra, B. The cosmological constant and dark energy. Reviews of Modern Physics. 75 (2): 559-606 (2003)

15. Uzan, J.-P. Varying constants, gravitation and cosmology. Living Reviews in Relativity. 14(1): 2 (2011)

16. Nemiroff, R. J., Patla, B. Adventures in Friedmann cosmology: A detailed expansion of the cosmological Friedmann equations. American Journal of Physics. 76 (3): 265-276 (2008)

17. Linde, A. Inflationary Cosmology after Planck 2013. (2014) arXiv:1402.0526v2 
18. Blumenthal, G. R.; Faber, S. M.; Primack, J. R.; Rees, M. J. Formation of galaxies and large-scale structure with cold dark matter. Nature. 311 (517): 517-525 (1984)

19. Maeder, A. et al. (Planck Collaboration) (2020). "Planck 2018 results. VI. Cosmological parameters". Astronomy \& Astrophysics. 641: A6

20. Jackson, N. The Hubble constant. Living Review Relativity 18, 2 (2015). https://doi.org/10.1007//rr-2015-2

21. Riess, A. G. et al. Observational Evidence from Supernovae for an Accelerating Universe and a Cosmological Constant. The Astronomical Journal. 116 (3): 10091038 (1998) arXiv:astro-ph/9805201.

22. Perlmutter, S. et al. Measurements of Omega and Lambda from 42 high redshift supernovae. Astrophysical Journal. 517 (2): 565-586 (1999)

23. Hahn, O., Porciani, C., Carollo, M, Dekel, A. Properties of dark matter haloes in clusters, filaments, sheets and voids, Monthly Notices of the Royal Astronomical Society, 375, 489-499 (2007) https://doi.org/10.1111/i.13652966.2006.11318.x 